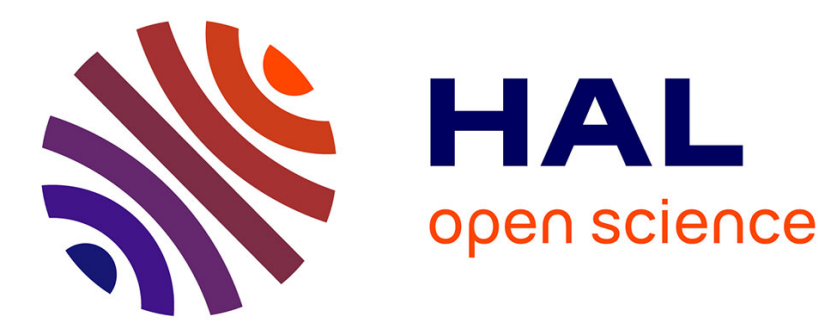

\title{
RE- et les différentes manifestations de l'itérativité
}

Denis Apothéloz

\section{To cite this version:}

Denis Apothéloz. RE- et les différentes manifestations de l'itérativité. Pratiques: linguistique, littérature, didactique, 2005, 125-126, pp.48 - 71. hal-02508917

\section{HAL Id: hal-02508917 \\ https://hal.univ-lorraine.fr/hal-02508917}

Submitted on 16 Mar 2020

HAL is a multi-disciplinary open access archive for the deposit and dissemination of scientific research documents, whether they are published or not. The documents may come from teaching and research institutions in France or abroad, or from public or private research centers.
L'archive ouverte pluridisciplinaire $\mathbf{H A L}$, est destinée au dépôt et à la diffusion de documents scientifiques de niveau recherche, publiés ou non, émanant des établissements d'enseignement et de recherche français ou étrangers, des laboratoires publics ou privés. 
Paru dans Pratiques, n ${ }^{\circ} 125-126,2005$, pp. 48-71.

\title{
$R E$ - et les différentes manifestations de l'itérativité
}

\author{
Denis Apothéloz \\ Université de Nancy 2, ATILF (UMR 7118)
}

\section{Préambule}

Cet article voudrait présenter un panorama des principaux faits et problèmes associés à la préfixation en $R E-$, et peut donc être lu comme une petite étude monographique. Du point de vue sémantique, l'une des hypothèses qui sous-tend nos analyses est que le schème dérivationnel marqué par cette préfixation est corrélé à une seule et unique valeur, en dépit des multiples effets de sens qu'il produit dans le lexique. Nous essayerons donc de montrer comment il est possible sans contradiction, tout à la fois d'adopter une conception unitaire de cette préfixation, et de rendre compte de la multiplicité des valeurs sémantiques qu'elle est susceptible de construire.

Du point de vue du lexique, l'un de ces effets de sens, qualifié ici d' " annulatif », est parfois lié à un genre particulier de relation d'antonymie, connu sous l'appellation d'antonymie directionnelle. Nous dégagerons les principales caractéristiques de cette relation et montrerons qu'elle produit, dans le lexique, des micro-classes de lexèmes structurées par deux effets sémantiques de $R E$ - et par deux types d'antonymie directionnelle.

Nous examinerons également le problème des variantes réalisationnelles de $R E-$, qu'il s'agisse d'allomorphes au sens habituel du terme ou d'autres phénomènes variationnels. De ce point de vue formel, nous verrons qu'il est possible de distinguer, en réalité, deux morphèmes $R E$-, contrastant aussi bien par leurs propriétés lexicales que par leurs propriétés morphophonologiques. A cet égard nous verrons qu'il y a une analogie de fonctionnement tout à fait frappante avec la préfixation négative en $I N-$. On évoquera à cette occasion la distinction bloomfieldienne entre affixe primaire et affixe secondaire.

Tout au long de cet article la convention suivante sera utilisée : la notation en petites capitales $(R E-)$ désignera le préfixe en tant qu'objet morphologique abstrait, indépendamment de ses différentes manifestations allomorphiques ; ces dernières seront notées en caractères bas de casse ou, si l'on préfère, minuscules (re-, ré-, $r$ - par exemple).

\section{Fait lexical et fait morphologique}

La plupart des travaux qui ont été consacrés à la préfixation en $R E$ - s'accordent pour constater la grande complexité de son fonctionnement sémantique et distinguent en général plusieurs valeurs (par exemple Léger 1956 ; Mok 1964, 1980 ; Jalenques 2001). Assez classiquement, ces approches polysémiques se trouvent cependant confrontées à deux problèmes principaux :

\footnotetext{
${ }^{1}$ Un chaleureux merci à Michel Roché (Université de Toulouse-Le Mirail), qui a lu une première version de cet article et m'a permis d'apporter de nombreuses améliorations.
} 
D'une part, à une multiplication pas toujours contrôlée des valeurs sémantiques identifiées, tendance particulièrement perceptible dans les travaux de lexicographie, qui se perdent parfois dans de longues énumérations de "nuances» de sens. Ces distinctions, souvent extrêmement fines et, pour cette raison, d'application difficile - quand ce n'est pas franchement subjective - masquent une éventuelle unité du phénomène étudié et aboutissent à des descriptions éclatées et peu cohérentes. On pourrait montrer par ailleurs que certaines d'entre elles attribuent à la préfixation des propriétés sémantiques dont l'origine se situe en réalité dans la base du dérivé, et non dans le préfixe lui-même.

L'autre problème que posent ces conceptions polysémiques de l'affixation est celui de la délimitation du phénomène étudié (problème classique en morphologie !), qui conduit aux inévitables questions du type : est-ce que des lexèmes comme réunir, renier ou reconnaître doivent être considérés, dans la synchronie actuelle, comme des dérivés en $R E-$ ? De fait, il est clair que ces questions ne sauraient trouver de réponse en dehors d'un cadre théorique, ne serait-ce que minimal, modélisant le phénomène étudié et donnant un statut explicite à la notion de mot construit et aux rapports entre morphologie et lexique. C'est donc par ce point que nous allons commencer.

\subsection{Le lexique comme ressource}

Commençons par rappeler que la propriété assurément la plus centrale du lexique est d'être une ressource d'unités préconstruites et mémorisées, prêtes à l'emploi (c'est-à-dire prêtes à entrer comme constituants dans des construits syntaxiques). Cette propriété est centrale en ceci qu'elle distingue massivement les construits lexicaux des construits syntaxiques, en dépit de toutes les réserves qu'on peut formuler à l'égard de cette frontière de niveaux (cf. Aronoff 1976). Linguistes et morphologues sont loin d'être d'accord sur le type des unités qui relèvent du lexique, notamment en raison de la différence entre unités grammaticales et unités lexicales, mais peu importe $\mathrm{ici}^{2}$. C'est sur cette qualité à la fois d'objet préconstruit et d'objet mémorisé - donc en tout temps " accessible » - que nous voudrions surtout insister. "Accessible » peut d'ailleurs s'entendre ici en deux sens différents : d'une part accessible à la mémoire, d'autre part accessible aux opérations syntaxiques.

$\mathrm{Au}$ sein de cette ressource, les lexèmes construits sont des unités dont la fabrication relève d'un schème de construction, lui-même mémorisé et disponible à titre de savoir-faire lexical, et étayé dans le lexique par une classe plus ou moins importante d'unités lexicales présentant le même pattern constructionnel. Ces schèmes sont en tout temps activables et fonctionnent aussi bien en «production » qu'en « reconnaissance » : d'une part, ils servent à construire des unités lexicales n'existant pas dans le lexique ; d'autre part, ils constituent un instrument constamment disponible pour l'analyse des unités existantes. Quand ils fonctionnent en reconnaissance, ils permettent par exemple aux usagers de la langue de reconnaître, et par conséquent d'interpréter sémantiquement, des lexèmes qui n'ont jamais été rencontrés auparavant.

\subsection{Caractère sédimentaire du lexique}

Outre sa dimension de ressource, le lexique présente également un caractère fondamentalement sédimentaire. On y rencontre, en effet, aussi bien des unités dont la structure interne est conforme aux schèmes de construction actuellement fonctionnels (lexèmes construits stricto sensu) que des « fossiles morphologiques », autrement dit des

\footnotetext{
${ }^{2}$ Voir Fradin (2003) sur des notions comme celle de « lexique restreint », « lexique étendu », etc., et pour une présentation critique de différentes conceptions et modélisations du lexique.
} 
unités complètement idiosyncratiques, dont la dimension construite ne présente plus aucune actualité et ne peut être appréhendée que dans une approche archéologique de la langue. Plusieurs causes peuvent être responsables de cette forme d'obsolescence : par exemple, une dérive du sens de l'unité construite par rapport au sens de ses composants morphologiques (de sorte que le sens de l'unité construite n'est plus compositionnel) ; ou l'obsolescence d'un composant morphologique : ainsi, l'obsolescence pratiquement complète de celer a pour conséquence que le verbe déceler n'est plus traitable comme un verbe construit de sorte qu'on peut dire qu'il est, littéralement, orphelin de sa base lexicale. Ces processus diachroniques sont souvent décrits comme des faits de lexicalisation, mais en un sens particulier de ce terme. Nous reviendrons plus loin sur cette notion centrale de lexicalisation.

De ces propriétés générales découle le fait suivant : une unité lexicale construite et faisant partie de la ressource lexicale (du lexique) peut être produite, en discours, et selon les circonstances, soit par accès mémoriel direct soit par construction (c.-à-d. par activation d'un schème constructionnel). Autrement dit, qu'une unité lexicale fasse partie du lexique mémorisé ne garantit pas que sa production ne sollicite pas un schème constructionnel.

Il convient donc de distinguer, au plan théorique, trois situations, correspondant à trois classes d'unités lexicales :

(1) les unités construites et présentes dans le lexique, auxquelles il est possible d'accéder soit mémoriellement soit constructionnellement ;

(2) les unités construites non présentes dans le lexique, auxquelles il n'est par conséquent possible d'accéder que par construction - Dell (1979) appelait ces unités « lexies homogènes »;

(3) les unités non construites et présentes dans le lexique, auxquelles il n'est possible d'accéder que mémoriellement.

Très schématiquement, (1) correspond aux mots construits faisant partie du lexique mental des usagers, (2) correspond à la néologie, et (3) aux lexèmes monomorphémiques et aux fossiles morphologiques. Ce point méritait d'être précisé, dans la mesure où l'on pourrait être tenté d'identifier, d'une part « lexème non construit » et « accès mémoriel », d'autre part « lexème construit » et « accès par activation d'un schème ».

L'une des difficultés auxquelles se trouve confrontée la morphologie constructionnelle est précisément cette hétérogénéité de la ressource lexicale, due à son caractère fondamentalement sédimentaire et archéologique ${ }^{3}$. Le problème type qui en découle, quand il s'agit d'analyser une unité, peut être formulé ainsi : l'unité considérée relève-t-elle seulement du lexique (cas 3 ci-dessus) ou relève-t-elle aussi de la morphologie (cas 1 ou 2) ? Il est clair que la réponse apportée à cette question ne saurait l'être sur des critères purement intuitifs, quand bien même le siège de ces intuitions serait un sujet parlant très informé du fait morphologique...

\subsection{Paraphrase du sens $V S$ modèle du sens}

Revenons à $R E-$ - Il a été dit plus haut que certaines descriptions tendent à en faire éclater la polysémie. Cependant un examen des analyses sémantiques généralement proposées conduit à réduire cette polysémie à deux classes principales d'effets de sens, qu'on appellera « sens itératif » et « sens annulatif ». Elles sont illustrées respectivement par des items comme recalculer, réopérer, redessiner (sens itératif), et redescendre, ressortir, recracher (sens annulatif). Intuitivement, le premier effet peut être restitué par une paraphrase comme " à nouveau » : " calculer à nouveau », " opérer à nouveau », " dessiner à nouveau »; le second est plus difficile à paraphraser mais on y trouve toujours l'idée d'annulation : redescendre,

\footnotetext{
${ }^{3}$ C'est là aussi une différence importante avec la syntaxe, où les phénomènes sédimentaires sont plus marginaux.
} 
" descendre après qu'on est monté "; ressortir, " sortir après qu'on est entré "; recracher, "cracher après qu'on a avalé ou mis dans sa bouche ".

L'expression d' « effet de sens » n'est pas choisie au hasard. Elle vise à suggérer qu'une certaine prudence est nécessaire quand il s'agit d'accorder un statut à une interprétation. Pour le dire en un mot, une paraphrase sémantique restituant une pure intuition, comme celles qui sont données ci-dessus, et telle qu'on en rencontre dans beaucoup de travaux en morphologie, ne saurait valoir pour une analyse du sens. Il nous paraît en effet essentiel de ménager un espace théorique entre nos intuitions interprétatives, telles que nous les exprimons habituellement au moyen de telles paraphrases, et les mécanismes sémantiques proprement dits. Il faut, en un mot, se garder de confondre paraphrase du sens et modèle du sens, comme le rappellent judicieusement Berrendonner \& Clavier (1997).

On notera que ces deux classes d'effets de sens n'ont pas tout à fait le même statut : les lexèmes qui manifestent un effet annulatif ont presque toujours (sinon toujours) aussi une acception itérative, l'inverse n'étant pas vrai (redescendre peut signifier aussi bien "descendre à nouveau »). D'où la question suivante : le sens annulatif est-il second par rapport au sens itératif ? Par ailleurs, du point de vue lexicologique, le sens annulatif est souvent corrélé à la relation d'antonymie et donc à la question de la négation dans le lexique (redescendre est un antonyme de monter). C'est un point sur lequel nous reviendrons.

\section{Modèle minimal}

\subsection{Le schème dérivationnel comme foncteur}

On utilisera ici la notion de foncteur pour décrire les lexèmes construits et les rapports qu'ils entretiennent avec leurs éléments constructeurs. La préfixation $R E$ - sera décrite comme l'application, à un argument, d'un foncteur lexical qu'on représentera par la formule « $\grave{a}$ nouveau $(x) »{ }^{4}$. Au plan du signifiant, ce foncteur laisse comme trace un segment (re-, ré- ou $r$-) correspondant à ce qu'on désigne habituellement par le terme de préfixe. Au plan du signifié, il produit un contenu sémantique consistant en une valeur d'itération appliquée à un argument, ce dernier pouvant être le (ou un) contenu de la base, ou un paramètre de ce contenu (ce point sera explicité plus loin). Selon nous, l'élucidation de la logique et des conditions qui déterminent la sélection de l'argument est l'une des questions centrales qu'il s'agit d'examiner, si l'on veut saisir unitairement le fonctionnement sémantique de $R E$ - et rendre compte de ses multiples effets de sens.

On ne fera ici qu'ébaucher cette analyse, nous bornant à présenter une liste non exhaustive de quelques cas censés exemplifier les principaux types d'arguments pouvant être sélectionnés par le foncteur. L'hypothèse qui est à la base de notre démarche est qu'il y a un unique foncteur $R E-$, monosémique, et que les divers effets de sens qu'il produit résultent seulement de la variation de sa " portée ». La position défendue ici reprend donc le principe des analyses présentées par Amiot (2002).

\subsection{Quelques cas types}

Prenons le verbe recalculer pour illustrer une première distinction. Considérons un énoncé minimal de la forme $A$ recalcule $X$. Trois interprétations de l'itérativité sont ici possibles. Dans la première, $\mathrm{A}$ calcule au moins pour la $2^{\mathrm{e}}$ fois $\mathrm{X}$; dans la deuxième, $\mathrm{X}$ est calculé au moins pour la $2^{\mathrm{e}}$ fois, mais l'agent de ce procès n'est pas le même que dans les

\footnotetext{
${ }^{4}$ Foncteur « lexical » parce que produisant comme résultat une unité lexicale. Cette notion de foncteur est empruntée aux logiciens de l’Ecole de Varsovie, en particulier à Ajdukiewicz (1967).
} 
occurrences antérieures du procès ; dans la troisième, A se livre au moins pour la $2^{\mathrm{e}}$ fois à l'activité de calcul, mais l'objet calculé n'est pas le même qu'antérieurement. Ces trois interprétations peuvent être discriminées par les présuppositions qu'elles impliquent sur l'état de l'univers représenté : la première interprétation présuppose validée l'information " $A a$ déjà calculé $X »$; la seconde présuppose validée l'information "il y a déjà eu calcul de $X$ »; la troisième présuppose validée l'information " $A$ s'est déjà livré à l'activité de calcul ». Ces trois interprétations peuvent être glosées par les formulations suivantes :

(i) «à nouveau A calcule $X$ »

(ii) "à nouveau il y a calcul de $X$ »

(iii) "à nouveau A calcule quelque chose "

La différence entre ces trois interprétations réside donc dans la sélection de l'argument du foncteur : dans (i) le foncteur porte sur " $A$ calcule $X$ », dans (ii) il porte uniquement sur l'événement que constitue le calcul de $X$, donc sur " il y a calcul de $X$ », et dans (iii) il porte sur " A calcule quelque chose ». Par commodité on désignera ces trois interprétations par les termes : (i) itération du procès, (ii) itération de l'événement, et (iii) itération de l'activité. En voici des illustrations :

(1) Il a calculé et recalculé plusieurs fois ce que ce voyage allait lui coûter.

(2) Tu t'es trompé ! Passe-moi la machine, je vais recalculer le total.

(3) La calculette est réparée, elle recalcule.

On pourrait dire, avec Amiot (2002), que dans (1) c'est toute la « scène actancielle » qui est répétée ; alors que dans (2) et (3) la scène actancielle n'est que partiellement répétée.

Un exemple de verbe qui a de fréquents emplois comme itération d'événement (type ii) est réexpédier, lorsqu'il s'agit de représenter des situations comme " $A$ a envoyé un message à $B$, qui l'a réexpédié à $C »$. Ce qui est réitéré ici, c'est l'expédition du message, indépendamment de l'identité de son expéditeur et de son destinataire.

Une deuxième distinction sera illustrée à partir du verbe redescendre dans ses emplois intransitifs. L'énoncé de la forme $A$ redescend a deux interprétations principales, qu'on peut formuler intuitivement comme : "A descend à nouveau » et " A descend pour aller là où il se trouvait avant qu'il soit monté $»^{5}$. Ces deux valeurs correspondent respectivement aux effets de sens itératif et annulatif mentionnés plus haut. L'interprétation itérative présuppose valide l'information " auparavant $A$ est descendu »; l'interprétation annulative présuppose valide l'information " auparavant A est monté ». Dans l'interprétation itérative, le foncteur porte sur " $A$ descend »; il appartient donc au type (iii). Dans l'interprétation annulative, il porte sur une proposition exprimant le lieu où se trouvera l'agent au terme de son mouvement de descente. Il s'agit là d'un quatrième type d'argument du foncteur. On peut représenter ces deux interprétations ainsi :

(iii) " à nouveau A descend"

(iv) " A descend dans le but d'être à nouveau au lieu où il se trouvait auparavant»

Les interprétations (iii) et (iv) distinguent les deux exemple suivants.

(4) Tu es déjà descendu à la cave il y a dix minutes. Et maintenant tu veux y redescendre. Qu'est-ce que tu vas faire dans cette cave ?

\footnotetext{
${ }^{5} \mathrm{D}$ 'autres valeurs de redescendre intransitif existent évidemment, non prises en considération ici.
} 
(5) Il a passé toute la journée au sommet de la colline et est redescendu en fin d'après-midi.

Nous dirons que le foncteur prend pour argument, dans le type (iv), un paramètre de descendre. Ce paramètre est la propriété consistant, pour l'agent de la descente, à se trouver au lieu-cible de son déplacement. Il s'agit donc de l'état résultatif associé au verbe base (descendre).

Dans leur acception annulative, des verbes comme remonter, ressortir, repartir, revenir, se relever, etc. peuvent être analysés de la même manière. D'autres verbes comme refermer, revendre ou recharger ont un fonctionnement identique, à ceci près que le paramètre concerne ici non pas l'agent mais l'objet: A referme $X$, au sens annulatif, peut être glosé comme " $A$ ferme $X$ de manière à ce que $X$ soit à nouveau fermé » ou " $A$ fait en sorte que $X$ soit à nouveau fermé ». Là aussi l'argument du foncteur n'est autre que l'état résultatif associé au verbe base (fermer).

Le type (iv) correspond à des valeurs que les lexicographes ont souvent décrites au moyen de gloses comme " retour au point de départ ", "retour à un état antérieur ", "retour en arrière », " mouvement dans une direction contraire à la direction initiale », etc. (voir par exemple Guilbert 1986, ainsi que le TLFi).

Revenons brièvement au cas de réexpédier. On s'aperçoit maintenant que certains emplois de ce verbe peuvent être décrits de deux manières différentes. Soit schématiquement l'exemple suivant : A expédie $X$ à $B$, qui réexpédie $X$ à $A$. Cet emploi de réexpédier, où certains lexicographes voient un $R E$ - à signification de réciprocité, peut être analysé soit comme relevant du type (ii) ("à nouveau il y a expédition de $X »$ ), soit comme relevant du type (iv) ("B expédie X dans le but que $X$ soit à nouveau en possession de $A$ »). On a peutêtre là une indication sur le rapport inférentiel, pragmatique, qu'il peut y avoir entre les effets de sens reçus comme itératifs et les effets de sens reçus comme annulatifs.

Examinons maintenant un verbe comme raccompagner, plus exactement un énoncé de la forme $A$ raccompagne $X$. Les interprétations (i), (ii) et (iii) décrites plus haut sont possibles. En effet cet énoncé peut être utilisé pour signifier l'une ou l'autre des trois valeurs itératives : itération du procès, itération de l'événement ou itération de l'activité ; soit :

(i) «à nouveau A accompagne $X$ »

(ii) " à nouveau il y a accompagnement de $X$ »

(iii) "à nouveau A accompagne quelqu'un"

Mais une autre interprétation de $A$ raccompagne $X$, probablement beaucoup plus fréquente, est celle qu'on pourrait paraphraser informellement par : " $A$ va avec X au lieu $Y$ où $X$ se trouvait auparavant $»$. De toute évidence l'itération ne porte ici ni sur le procès ni sur l'événement ni sur l'activité dénotés par accompagner. L'information présupposée valide est " auparavant $X$ a quitté un certain lieu $Y$ » ou " auparavant $X$ se trouvait au lieu $Y$ », de sorte que l'interprétation relève clairement de la classe des effets de sens annulatifs. Mais diffère-telle du type (iv) ci-dessus ? Pas fondamentalement. L'itération porte, comme dans le cas de refermer, sur l'état résultant associé à la base accompagner : à savoir un contenu qu'on peut gloser comme " $X$ se trouve au lieu $Y$ ». C'est ce contenu propositionnel qui constitue l'argument du foncteur "à nouveau $(x)$ ». L'argument du foncteur est donc ici encore un paramètre du verbe base. Ce que fait voir la glose :

(iv) « A accompagne $X$ en un lieu $Y$ de façon à ce que à nouveau $X$ soit au lieu $Y$ » 
Un verbe comme reconduire présente la même structure. Comme plus haut, on s'aperçoit que la différence entre ces interprétations tient seulement à la portée du foncteur (celle-ci étant elle-même dépendante de la valence du verbe). Le foncteur a pour argument " A accompagne $X$ » dans (i), " il y a accompagnement de $X$ » dans (ii), " A accompagne quelqu'un " dans (iii), et " $X$ se trouve au lieu $Y$ » dans (iv). Notons au passage que les variantes (i)-(iii) de l'itération seraient beaucoup plus probablement exprimées par des formes comme réaccompagner ou re-accompagner, que par raccompagner. On reviendra sur ce point essentiel dans la section 6 du présent article. Toutes ces interprétations sont illustrées par les exemples suivants :

(6) Hier je l'ai accompagné à la gare. Aujourd'hui je l'ai re-accompagné.

(7) Hier tu l'as accompagné à la gare. Aujourd'hui je l'ai re-accompagné.

(8) Hier je l'ai accompagné à la gare. Aujourd'hui je re-accompagne sa sœur.

(9) Il a passé la nuit chez moi. Le lendemain je l'ai raccompagné à la gare.

Une autre variation d'effet de sens, que nous nous bornerons à signaler rapidement, est celle qu'on observe en comparant recalculer et refonctionner. En effet, ces deux verbes ne construisent pas exactement la même représentation de l'itérativité. La différence peut être formulée de la façon suivante : alors que recalculer, quelle que soit sa valeur itérative (itération du procès, de l'activité ou de l'événement), construit la représentation de deux (au moins) procès successifs et distincts, refonctionner construit la représentation d'un seul et unique procès ayant subi une interruption. Cette différence tient, comme l'a noté Amiot (2002), aux propriétés aspectuelles respectives des bases de ces verbes (et par conséquent de ces verbes eux-mêmes) : calculer dénote un procès télique, c.-à-d. dont le déroulement conduit à un résultat et implique par conséquent une borne finale ; tandis que fonctionner dénote un procès non télique et continu, donc non intrinsèquement borné ${ }^{6}$.

Examinons enfin quelques cas qui ne peuvent pas être décrits en référence à la structure argumentale de la base verbale.

(10) [...] avant d'être mon meilleur ami, c'était mon copain, et puis on a cassé et c'est mon meilleur ami, et maintenant c'est re mon chéri. (Ecrit électronique)

(11) Miss contradiction, après ce passage survolté, nous emmène (seule sur scène avec son piano) sur sa plus belle ballade (à mes yeux du moins), « Sur toi », et là c'est re-émotions, re-briquet... un très beau moment. (Ecrit électronique)

(12) Je sais seulement que Mme Daudet est re-mère. (Flaubert, Corrresp., 1878, 133. < Frantext)

(13) Solange a froid, chaud, re-froid, mal au poignet, mal au cœur [...]. (C. de Rivoyre, Les sultans, 21. < Frantext)

(14) Resalut!

Apparemment le point commun de ces exemples est que la préfixation $R E$ - n'y opère pas sur une forme verbale mais sur un nom, un syntagme nominal ou un adjectif. Toutefois, quand il s'agit d'un nom ou d'un syntagme nominal, ils ont généralement un statut sémantique

\footnotetext{
${ }^{6}$ De façon générale toutes ces observations indiquent que la préfixation $R E$ - se caractérise par le fait qu'elle induit des représentations sémantiques impliquant un véritable scénario d'événements, et qu'elle interagit de façon complexe avec les phénomènes aspectuels. Voir sur ce point Mascherin (à paraître).
} 
particulier. Dans (10), l'expression mon chéri est une apostrophe, plus exactement ici une apostrophe rapportée ; c'est cette apostrophe qui constitue l'argument du foncteur « $\grave{a}$ nouveau $(x)$ ». Contrairement aux apparences, ce n'est donc pas sur un syntagme nominal que porte ici le foncteur mais sur une énonciation mentionnée. Ce cas peut être assimilé à une itération d'événement (type (ii)). Dans (11), émotions et briquet ne sont pas des désignations d'objets mais sont employés comme des phrases nominales, leur valeur sémantique étant celle d'une proposition présentative (" il y a des émotions", " il y a des briquets qui sont allumés »). Ce sont ces propositions présentatives qui constituent l'argument du foncteur. Il s'agit donc encore d'une itération de type (ii). Dans (12), RE- porte sur la prédication être mère, et dans (13) sur avoir froid. Quant à (14), il s'apparente à (10) en ceci que le foncteur y a pour argument une énonciation (une occurrence antérieure d'un acte de salutation). A vrai dire tous ces emplois de $R E$ - s'apparentent fortement à des adverbes.

On voit qu'il est erroné d'inférer de la forte affinité qu'on observe entre $R E$ - et la catégorie des verbes, que ce schème constructionnel ne s'applique qu'à des verbes. Plus juste serait de dire qu'il implique des valeurs processuelles ou « actionnelles ».

Nous arrêterons là cet inventaire, qui ne peut être qu'incomplet dans le cadre de cet article. Essayons d'en tirer quelques principes généraux pour le problème signalé plus haut de la frontière entre unité construite et unité non construite.

\section{Construit $v S$ non construit}

Les analyses qui viennent d'être esquissées semblent apporter une confirmation à l'hypothèse monosémique formulée au $\S 2.1$. Ainsi sommes-nous fondés à exclure de la classe des construits en $R E$ - tout item dont le sens ne comporte pas, à un niveau ou à un autre de sa structure sémantique, le foncteur « à nouveau $(x)$ ».

Cependant l'existence même de ce foncteur est tributaire de celle d'un argument sur lequel il puisse «porter ». Cet argument doit être accessible à partir du sens de la base de la dérivation, qu'il s'agisse de la base elle-même ou d'un paramètre de celle-ci. Nous avons vu que ces paramètres ont systématiquement à voir avec un argument de la base (un complément), ce qui n'a rien d'étonnant si l'on songe que $R E$ - est un préfixe intimement lié aux valeurs processuelles et actionnelles.

Enfin il faut que la relation sémantique entre le construit et sa base soit une relation d'hyponymie (le construit étant un hyponyme de la base).

En résumé, il faut que le sens du lexème supposé construit (1) comporte, à quelque niveau que ce soit de sa structure sémantique, un composant " à nouveau », (2) que ce composant trouve son argument dans la structure sémantique de la base, et (3) que le construit soit dans une relation d'hyponymie par rapport à sa base.

Nous sommes maintenant en mesure de préciser la distinction entre lexème construit et lexème non construit, concernant les supposés dérivés en $R E-$. Tout lexème ne satisfaisant pas les trois critères ci-dessus sera réputé sans rapport avec notre foncteur et donc, de ce point de vue, non construit. Considérons par exemple un verbe comme raccommoder. Il est possible de mettre au jour un composant " à nouveau » dans le sens de ce lexème, comme le suggèrent des paraphrases comme "faire subir des modifications à $X$ de façon à ce que $X$ se trouve à nouveau dans l'état où il se trouvait antérieurement », etc. Le critère (1) paraît donc satisfait. Intuitivement, il semble d'ailleurs qu'on puisse bel et bien analyser raccommoder comme un verbe exprimant l'idée d'annulation. La paraphrase ci-dessus indique que le critère (2) est également satisfait : on peut en effet considérer que le foncteur trouve ici son argument dans un paramètre du sens du verbe base accommoder. Mais le troisième critère fait difficulté : accommoder ( adapter ») et raccommoder entretiennent une relation non pas d'hyponymie 
mais, au plus, de co-hyponymie, leur hyperonyme commun (s'il fallait leur en trouver un) étant un verbe comme modifier ou transformer. Pour cette raison il faut exclure raccommoder de la classe des items construits au moyen du foncteur « à nouveau $(x)\rangle^{7}$. On peut en dire autant de réunir. Ce verbe doit aussi être exclu de la classe des items construits au moyen de notre foncteur, car son sens ne comporte aucune espèce d'idée d'itération ${ }^{8}$.

\section{Morphémisation d'un composant sémantique}

L'hypothèse d'une préfixation $R E$ - unique et monosémique est confirmée par une autre observation. Il s'agit du fait suivant, repéré par les lexicographes mais malheureusement décrit la plupart du temps comme un simple fait de " renforcement».

On s'est avisé depuis longtemps de l'existence de couples de verbes dont la forme indique qu'ils sont dérivationnellement reliés par $R E-$, mais dont le sens, du moins dans une partie de leurs emplois, est pratiquement indiscriminable (ce qui ne signifie pas, bien entendu, qu'il ne contraste pas dans d'autres emplois). En voici un petit échantillon :

(15) (a)

doubler

copier

traduire

transcrire

ajouter

actualiser

couvrir

allonger (b)

redoubler

recopier

retraduire

retranscrire

rajouter

réactualiser

recouvrir

rallonger

Ces sortes d'équivalences sémantiques donnent lieu, c'est bien connu, à diverses hésitations : copier (ou recopier) une lettre?, doubler (ou redoubler) son année de licence?, couvrir (ou recouvrir) d'une couche de farine ?, ajouter (ou rajouter) une pincée de sel ? etc. Décrire ces verbes en $R E$ - comme des formes sémantiquement ou formellement renforcées, comme on le fait parfois, est évidemment peu satisfaisant, cette notion de renforcement étant des plus floues ${ }^{9}$.

Quoi qu'il en soit, à examiner d'un peu plus près la liste ci-dessus, on s'aperçoit que les verbes de la colonne (a) comportent intrinsèquement un composant de sens itératif. Tel est le cas de doubler, copier, traduire, transcrire, ajouter, actualiser, et dans une moindre mesure

\footnotetext{
${ }^{7}$ Il est toutefois possible que la présence d'un composant sémantique " à nouveau », sanctionnée par $r$ - ou $r a$-, conduise les usagers à voir dans ce segment un marqueur de classe (Michel Roché, com. pers.).

${ }^{8}$ Les sujets parlants montrent parfois, par divers jeux de langage, qu'ils perçoivent bien la différence entre les fossiles morphologiques et les lexèmes réellement construits. Voir ces exemples, trouvés dans la base Frantext : S'il est récréation, le théâtre est aussi re-création (M.-T. Serriere, Le TNP et nous, 173); Si au lieu de nous reconnaître, nous nous re-connaissions (Corresp. Gide-Valery, 1942, 472). Ce type de jeu de langage est également un indice du fonctionnement " analytique », et pas seulement " génératif », des schèmes de construction.

${ }^{9}$ Le problème est notamment de savoir si, par « renforcement », on veut désigner un fait de pure forme ou un fait sémantique. Par exemple, le fait que la forme latine de la négation préverbale non ait été complétée par la forme postverbale passu (d'où en français le morphème discontinu ne...pas) est souvent décrit comme relevant d'un mécanisme de renforcement (cf. Lehmann 1995). Toutefois la fonctionnalité de ce type de renforcement relève essentiellement, nous semble-t-il, de la saillance des morphèmes en tant que formes matérielles. C'est d'ailleurs pour cette absence de fonctionnalité sémantique que ces "procédés » ont souvent été attaqués par certains puristes, qui y voyaient une pure redondance. Tout autre est évidemment l'hypothèse que nous proposons pour les exemples (15b).
} 
couvrir, allonger. On est donc amené à formuler l'hypothèse que l'origine des doublons de la colonne (b) n'est autre que le sémantisme même du verbe base : la forme en $R E$ - résulterait ainsi d'un marquage secondaire, par un segment morphologique, d'une valeur d'itération interne au verbe base. Nous appellerons « morphémisation » ce marquage.

Il est important de noter que la morphémisation ne produit pas à strictement parler une forme construite (ou alors il s'agit d'une forme construite d'un genre particulier) ; elle ne fait en réalité que rendre manifeste, par un segment affixoïde, un composant sémantique déjà présent dans la base. Les formes lexicales résultant de ce phénomène devraient par conséquent être soigneusement distinguées de celles résultant d'un schème constructionnel authentique!

De la même manière, il semble que les verbes dont le sens implique un composant annulatif tendent eux aussi, quoique de façon moins nette, à développer ce type de doublon sémantique. Telle est peut-être l'explication de couples comme approvisionner et réapprovisionner, charger et recharger, coiffer et recoiffer, laver et relaver (dans certaines régions on parle de poudre à relaver, etc.) : le premier et le second termes de ces couples ont certains emplois ici aussi parfaitement interchangeables ${ }^{10}$.

Ce phénomène de morphémisation est voisin de ce que Corbin (1989) a appelé « intégrateurs paradigmatiques ». Un exemple classique en est le mot peuplier. En ancien français, le nom du peuplier (l'arbre) est peuple. Or ce nom a été modifié par ajout du segment -ier, non pas pour construire le nom de l'arbre (celui-ci était justement peuple) mais pour aligner la forme du mot sur le paradigme des dérivés en -ier, qui comprend justement de nombreux noms d'arbres et de végétaux (poirier, pommier, rosier, etc.). Il en résulte que le segment -ier dans peuplier est purement analogique et n'a aucune fonction sémantique ; en revanche il intègre formellement le nom de cet arbre dans un paradigme - d'où l'appellation d' « intégrateur paradigmatique ».

Dans le même ordre d'idée on pourrait mentionner d'autres phénomènes, comme la pression à la remotivation, que subit un verbe comme rabâcher. La syllabe initiale $r a$ de ce verbe, qui n'a selon le Robert historique rien à voir étymologiquement avec le préfixe $R E$ - (ce verbe a signifié anciennement « faire du tapage »), est fréquemment réinterprétée comme une instance de ce préfixe, en raison du contenu sémantique actuel du verbe (dans lequel la notion de répétition est centrale). D'où la transformation fréquente de $r a$ en $r e$. Les deux exemples ci-dessous en sont une attestation :

(16) On nous rebâche sans arrêt les oreilles avec ce terme qui fait froid dans le dos : cellulite ! (écrit électronique)

(17) Ironiquement, je dirais que l'expatriation présente un avantage principal, celui de l'oubli... L'oubli de la France et de son climat absolument déprimant, du rebâchage continuel sur la crise et l'absence de postes. (écrit électronique)

D'autres doublons ou quasi-doublons en $R E$ - mériteraient d'être examinés à la lumière de cette hypothèse, comme courber et recourber, grouper et regrouper, par exemple. Au total on constate que les formes en $R E$ - présentent une grande diversité de phénomènes, au sein desquelles la distinction entre les formes issues d'un schème constructionnel et les autres formes est des plus délicates.

\footnotetext{
${ }^{10}$ On sait qu'au plan théorique, certains modèles de la morphologie constructionnelle considèrent qu'il faut dissocier le marquage morphologique des opérations constructionnelles proprement dites (cf. par exemple Beard 1990). Le phénomène dont il est question ici est évidemment un argument important en faveur d'une telle conception.
} 


\section{Entracte lexicologique : $R E$ - et la relation d'antonymie}

Quittons un instant ces considérations morphologiques pour nous pencher sur les conséquences de la valeur annulative, telle qu'elle est construite par $R E-$, sur l'organisation du lexique. Il a été dit plus haut que $R E$ - est lié à l'antonymie. Sur ce point deux observations doivent être faites.

La première est qu'un nombre important de construits en $R E$ - à valeur annulative entrent comme terme dans un couple d'antonymes. Ces couples actualisent presque toujours un type d'antonymie que Lyons (1977) a qualifié de « directionnelle ».

(18) monter / redescendre, entrer / ressortir, éteindre / rallumer, arriver / repartir, perdre / retrouver, disparaître / réapparaître, etc.

La deuxième observation est que cette même relation d'antonymie concerne également la base du dérivé en $R E-$. D'où, en réalité, une double série d'antonymes :

(19) (a)

monter / descendre

entrer / sortir

éteindre / allumer

arriver / partir

perdre / trouver

disparaître / apparaître

etc. (b)

monter / redescendre

entrer / ressortir

éteindre / rallumer

arriver / repartir

perdre / retrouver

disparaître / réapparaître

On a là un phénomène qui intéresse la logique même de l'organisation du lexique, telle qu'elle peut se manifester à travers l'existence de micro-systèmes lexicaux. Examinons successivement ces deux points.

\subsection{L'antonymie directionnelle}

La question que nous voudrions poser est celle de savoir si le rapport d'antonymie est le même dans les couples (19a) et (19b) ci-dessus. Commençons par observer que d'autres schèmes constructionnels concernent l'antonymie en français. En particulier $I N-$ et $D E-$. Le premier est lié à l'expression de rapports comme la contradiction (pair / impair) ou la contrariété (confortable / inconfortable) ; le second est lié, comme $R E$-, à l'antonymie directionnelle : faire / défaire, réglementer / déréglementer, stabiliser / déstabiliser, crocher / décrocher, etc. ${ }^{11}$ Cependant, tandis que $I N$ - et $D E$-peuvent être qualifiés de préfixations " négatives », il n'en va pas de même pour $R E$-, en dépit de ses affinités avec l'antonymie. La raison en est la suivante.

$I N$ - et $D E$ - construisent systématiquement un item lexical antonyme de leur base : inconfortable est un antonyme (de type " contraire ») de confortable, défaire est un antonyme (de type « directionnel ») de faire, etc. Si on considère que la relation d'antonymie a quelque chose à voir avec la négation, alors on peut admettre que $I N$ - et $D E$ - sont des morphèmes négatifs. Mais tout autre est le cas de $R E$-, qui ne construit pas à proprement parler des antonymes : redescendre, dans sa valeur annulative, n'est pas un antonyme de descendre (mais de monter), ressortir dans sa valeur annulative n'est pas un antonyme de sortir (mais d'entrer), rallumer dans sa valeur annulative n'est pas un antonyme d'allumer (mais

\footnotetext{
${ }^{11}$ Mais pas de façon exclusive à ce type d'antonymie : cf. déplaire.
} 
d'éteindre), etc. $R E$ - n'est donc producteur d'antonyme qu'indirectement : il ne l'est que dans la mesure où sa base entre elle-même déjà dans un rapport d'antonymie avec un autre lexème. Le lexème qu'il construit ne fait alors que « dédoubler » ce rapport d'antonymie déjà existant, d'où des doubles couples comme (19). Mais si la base n'a pas d'antonyme lexicalisé, le dérivé en $R E$ - n'est antonyme d'aucun lexème. Tel est par exemple le cas d'un verbe comme ranimer : son sens (du moins l'un de ses sens) est indéniablement annulatif, mais il n'entre pas dans un rapport d'antonymie avec un autre verbe (la position avec laquelle il pourrait entrer dans ce type de rapport n'est pas lexicalisée) ; tel est également le cas de reconduire, ou de réteindre, qui n'a qu'un sens itératif et ne saurait être un antonyme directionnel de allumer.

Cependant, est-ce à dire pour autant que $R E$ - construise ce qu'il est convenu d'appeler un doublon ? La réponse est évidemment « non »- pour autant bien sûr qu'on admette que descendre et redescendre annulatif ne sont pas synonymes, pas davantage que sortir et ressortir annulatif, allumer et rallumer annulatif, etc. En quoi, par conséquent, la relation entre monter et descendre est-elle différente de celle entre monter et redescendre ? ou la relation entre entrer et sortir, différente de celle entre entrer et ressortir ? etc.

Notons tout d'abord que chacun des couples de (19) satisfait bien à la définition de l'antonymie directionnelle : on est chaque fois en présence de deux prédicats verbaux dénotant des procès de type « changement d'état », l'un des termes pouvant être schématisé par la formule $E_{i} \rightarrow E_{j}$ (transformation de l'état $E_{i}$ en l'état $E_{j}$ ), l'autre par la formule $E_{j} \rightarrow E_{i}$. L'opposition de directionnalité réside précisément dans cette idée de réversibilité d'un processus transformateur ${ }^{12}$. Il semble donc que la différence entre les séries (19a) et (19b) doive être caractérisée à l'intérieur même de la directionnalité.

On pourrait considérer qu'il existe en réalité deux type d'antonymies directionnelles. Le premier consiste en une opposition purement lexicale. Les termes liés par cette opposition le sont seulement en vertu de leur signifié (qu'on peut décrire, rappelons-le, comme une transformation). Les couples listés sous (19a) relèvent de cette antonymie directionnelle purement lexicale. Il n'y a à proprement parler ici aucune valeur annulative.

Le second type d'antonymie directionnelle est une conséquence de la valeur annulative associée à certains dérivés en $R E$-. Ceux-ci présentent en effet la particularité de présupposer l'accomplissement préalable d'un procès spécifique. Plus exactement, leur emploi en discours revient à valider, dans l'univers du discours, une information qu'on peut formuler analytiquement comme suit ${ }^{13}$ :

(20) " un procès transformateur a eu lieu, qui est directionnellement inverse de celui dénoté par le verbe base»

C'est ainsi que tout emploi de redescendre annulatif présuppose validée, dans l'univers du discours, l'information " un procès transformateur a eu lieu, qui est directionnellement inverse de celui dénoté par le verbe 'descendre’ » (i.e. celui que lexicalise le verbe monter); tout emploi de ressortir annulatif présuppose validée, dans l'univers du discours, l'information " un procès transformateur a eu lieu, qui est directionnellement inverse de celui dénoté par le verbe 'sortir' » (i.e. celui que lexicalise le verbe entrer); etc. Ce genre de présupposition fait donc des dérivés en $R E$ - annulatifs des sortes de lexèmes intrinsèquement anaphoriques ! Pour cette raison, on désignera les couples d'antonymes réunis sous (19b) comme des antonymes discursifs.

\footnotetext{
${ }^{12}$ Que ces changements d'état impliquent tantôt l'espace, comme monter / redescendre, tantôt non, comme perdre / retrouver, est ici secondaire. La directionnalité doit bien sûr être comprise indépendamment de toute référence spatiale.

${ }^{13}$ La glose (20) vaut pour les dérivés en $R E$ - à sens annulatif et ayant un antonyme lexicalisé. Des items comme raccompagner ou reconduire ne satisfont pas la deuxième condition (ils n'ont pas d'antonyme lexicalisé).
} 
On observera que l'opposition, dans les antonymes directionnels lexicaux (19a), est en quelque sorte atemporelle. En revanche, dans les antonymes directionnels discursifs (19b), elle implique nécessairement une certaine temporalité. C'est que les antonymes discursifs en $R E$ - engendrent une représentation sémantique consistant en un véritable scénario d'événements.

\subsection{Micro-systèmes lexicaux}

Cependant, pour des raisons qui restent d'ailleurs à élucider, les deux dérivés en $R E$ d'un couple d'antonymes directionnels lexicaux, comme (19a), ne lexicalisent pas toujours une valeur annulative. D'où l'existence de deux micro-systèmes lexicaux caractérisés par les propriétés suivantes :

- Dans le premier, chacun des termes du couple d'antonymes directionnels lexicaux (type a) a aussi un antonyme directionnel discursif en $R E$ - (type b, donc à valeur annulative). Il en résulte un micro-système d'antonymes composé de 4 lexèmes reposant sur 2 bases, organisés en 3 paires d'antonymes directionnels ( 1 paire d'antonymes directionnels lexicaux, 2 paires d'antonymes directionnels discursifs). Voici quelques séries réalisant ce type de micro-système.

(21) antonymes lexicaux monter / descendre entrer / sortir acheter / vendre baisser / lever ouvrir / fermer se lever/se coucher donner / prendre importer / exporter antonymes discursifs monter / redescendre entrer / ressortir acheter / revendre baisser / relever ouvrir / refermer se lever/se recoucher donner / reprendre importer / réexporter antonymes discursifs descendre / remonter sortir / r(e)entrer vendre / racheter $^{14}$ lever / rebaisser fermer / r(e)ouvrir se coucher/se relever prendre / redonner exporter / réimporter

- Dans le deuxième micro-système, l'un des termes seulement du couple d'antonymes directionnels lexicaux a un antonyme directionnel discursif. L'autre peut avoir développé un dérivé en $R E-$-, mais ce dernier n'a pas lexicalisé de valeur annulative. D'où un micro-système d'antonymes composé 3 lexèmes reposant sur 2 bases, organisés en 2 paires d'antonymes directionnels. (Le dérivé en $R E$ - à valeur purement itérative, n'entrant pas dans un rapport d'antonymie, est signalé ci-dessous entre parenthèses dans la $3^{\mathrm{e}}$ colonne.) Relativement aux séries listées en (21), ce micro-système apparaît donc lexicalement défectif.

(22) antonymes lexicaux disparaître / apparaître perdre / trouver éteindre / allumer arriver / partir recevoir / envoyer défaire / faire détruire / construire décoiffer / coiffer dégonfler / gonfler déchausser / chausser

\section{antonymes discursifs} disparaître / réapparaître perdre / retrouver éteindre / rallumer arriver / repartir recevoir / renvoyer défaire / refaire détruire / reconstruire décoiffer / recoiffer dégonfler / regonfler déchausser / rechausser pas de valeur annulative

(redisparaître)

(reperdre)

(réteindre)

(re-arriver)

(re-recevoir)

(redéfaire)

(redétruire)

(redécoiffer)

(redégonfler)

(redéchausser)

\footnotetext{
${ }^{14}$ Acheter et vendre sont en réalité des antonymes converses.
} 
La différence entre (21) et (22) indique peut-être que l'opposition de directionnalité des antonymes lexicaux est reçue comme symétrique dans (21), et comme non symétrique, ou orientée, dans (22). Se lever et se coucher dénoteraient ainsi des changements d'état parfaitement symétriques, du moins représentés comme tels ; tandis que coiffer et décoiffer dénoteraient des changements d'état qu'on pourrait dire orientés, en ce sens que l'un des deux états (être coiffé) y serait reçu comme plus « normal » que l'autre. Cette hypothèse est étayée par le fait que les valeurs annulatives sont assez typiquement associées à l'idée de " retour à l'état normal ». Elle expliquerait que recoiffer lexicalise une valeur annulative, mais non redécoiffer. On notera par ailleurs que les antonymes directionnels lexicaux de (22) ont souvent l'un de leur terme préfixé en dé-. Il y a là peut-être un indice supplémentaire de ce phénomène d'orientation ${ }^{15}$.

Ces analyses montrent que la propagation d'un schème constructionnel dans le lexique (se traduisant par une plus ou moins grande vitalité, etc.) n'est pas affaire que de « besoins communicationnels », de « pertinence référentielle » et autres facteurs pragmatiques. Elle est aussi solidaire de la constitution de sous-classes lexicales délimitées par quelques principes relationnels en assurant la cohésion et la reproductibilité.

\subsection{Remarques sur la notion de lexicalisation}

On notera, pour en terminer sur ce point, que nous avons utilisé ici le terme de « lexicalisation » dans un sens particulier. Rappelons que ce terme est susceptible de désigner au moins quatre processus diachroniques distincts (cf. Apothéloz 2002). Soit :

(a) la diminution ou la perte de la compositionnalité sémantique ;

(b) le codage d'un signifié par une forme lexicale ;

(c) l'émergence d'une forme lexicale à partir d'une forme appartenant à une autre catégorie ;

(d) l'intégration d'une forme construite dans la ressource lexicale des usagers.

Relève de la lexicalisation au sens (a) un lexème comme désaltérer. La perte de la compositionnalité sémantique de ce verbe tient au fait que sa base altérer est devenue complètement obsolète dans les emplois où elle signifiait "donner soif ». Plus proche de notre problème est le cas des verbes rassurer ou remplir. Comme désaltérer, rassurer a une base assurer qui existe bel et bien, mais dont le sens est tel que rassurer n'est plus sémantiquement compositionnel. Quant à remplir, il est lui aussi devenu pratiquement monomorphémique par obsolescence de sa base emplir. La lexicalisation au sens (a) correspond à ce qu'on appelle parfois aussi « démotivation ».

Relève de la lexicalisation au sens (b) ce qui a été dit plus haut à propos par exemple de ranimer : en dépit d'une évidente valeur annulative, ce verbe n'a pas d'antonyme lexicalisé. Il en va de même pour les dérivés en $R E$ - de la $3^{\mathrm{e}}$ colonne de (22), qui n'ont pas lexicalisé de valeur annulative, mais seulement une valeur itérative.

Relèvent de la lexicalisation au sens (c) des lexèmes nominaux comme carte bleue ou $q u$ 'en-dira-t-on, dont l'origine est respectivement un syntagme nominal et une proposition interrogative.

Relèvent enfin de la lexicalisation au sens (d) les phénomènes auxquels nous faisions allusion au début de cet article à propos du lexique comme ressource : il ne fait pas de doute que certaines formes construites, à un moment donné de leur histoire, sont en quelque sorte

\footnotetext{
${ }^{15}$ Ces observations nous ont été suggérées par M. Roché (com. pers.).
} 
« entièrement nouvelles » en ce sens que leur production relève toujours d'un mécanisme de construction et jamais de rappel mémoriel (pour la simple raison qu'elles ne font pas partie du répertoire lexical des usagers). Le processus au terme duquel une unité de ce type en vient à être progressivement mémorisée et intégrée dans la ressource lexicale est précisément ce que désigne le terme de lexicalisation au sens (d).

\section{Allomorphies et autres phénomènes variationnels}

Revenons maintenant à des problèmes spécifiquement morphologiques, plus particulièrement à la variabilité des manifestations de $R E$ -

\subsection{Allomorphies}

$\mathrm{Du}$ point de vue de ses allomorphies, $R E$ - se manifeste segmentalement par re(ressortir), ré- (réinventer), $r$ - (rouvrir) et ra-(rajeunir). Les régularités distributionnelles qui se dégagent sont à peu près les suivantes : la variante re- est sélectionnée quand la base a une initiale consonantique ; la variante ré-, quand la base a une initiale vocalique ${ }^{16}$; et la variante $r$ - quand la base commence par l'une des voyelles $\{[\mathrm{a}],[\tilde{\mathrm{a}}],[\varepsilon],[\mathrm{e}],[\mathrm{u}]\}$. Il y a donc une importante intersection entre les environnements des variantes ré- et $r$ - : cf. $r+$ acheter et

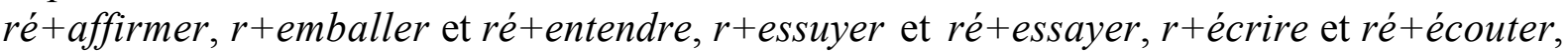
$r+o u v r i r$ et $r e ́+o u b l i e r$. Cette situation donne d'ailleurs lieu à des doublons : $r+e ́ c r i r e ~ e t$ ré+écrire, $r+e s s a y e r$ et ré+essayer, $r+$ ouvrir et ré+ouvrir, etc. - Quant à la variante $r a-$, elle est complètement obsolète (voir toutefois quelques exemples de formations régionales analysés par Roché, à paraître $)^{17}$.

Cependant cette distribution présente elle-même beaucoup de variation, tant au plan des allomorphies qu'au plan des valeurs sémantiques construites. Par exemple, il semble que l'intersection des environnements de ré- et de $r$ - signalée ci-dessus soit partiellement associée à une différenciation sémantique quand il y a doublon morphologique (Mok 1980) : le construit en $r$-tendrait à manifester une valeur itérative et une valeur annulative, tandis que le construit en ré- ne manifesterait que la valeur itérative : cf. des couples comme se rasseoir et se réasseoir, rapporter et réapporter, récrire et réécrire. A ce type de difficulté s'ajoute le fait que, ce schème constructionnel étant extrêmement productif, il est des plus difficile d'en délimiter les productions lexicales, dont beaucoup ne sont pas répertoriées par les lexicographes, bien que d'un usage courant et donc lexicalisées au sens (d) ci-dessus.

C'est cependant un autre phénomène que nous allons examiner maintenant. Il est lui aussi lié à un processus de différenciation sémantique, mais sa manifestation est beaucoup plus systématique que la différenciation ré- / r- évoquée ci-dessus. Un premier exemple fera voir de quoi il s'agit.

\footnotetext{
${ }^{16}$ Les lexèmes présentant la séquence ré- + consonne sont un peu plus d'une vingtaine et leur compositionnalité est presque toujours extrêmement douteuse : cf. récompenser, réconcilier, réconforter, récurer, régurgiter, réformer, réjouir, réveiller, réviser. Est en revanche indiscutablement construit au sens des critères établis plus haut le verbe réchauffer, dont le sens est exclusivement annulatif.

${ }^{17}$ Cette affirmation doit toutefois être tempérée par les phénomènes de métanalyse (au sens de Jespersen 1922). En présence de familles dérivationnelles comme mener, amener, ramener; crocher, accrocher, raccrocher; baisser, abaisser, rabaisser, il est vraisemblable que les sujets parlants analysent le troisième terme de ces triplets tantôt comme comportant un préfixe $r$-, tantôt comme comportant un préfixe ra- ( $r$-amener VS ra-mener, etc.). Cette situation se présente également avec certains dérivés déadjectivaux, comme raccourcir, rafraîchir, rajeunir, ralentir, ramollir, rapetisser, dont l'analyse présente encore d'autres problèmes que nous ne pouvons évoquer ici faute de place.
} 
Soit le couple d'antonymes directionnels (lexicaux) (se) déshabiller / ( $s^{\prime}$ )habiller. Il appartient au deuxième type de micro-système décrit plus haut. En effet, il est associé à un deuxième couple d'antonymes directionnels, discursif celui-là, construit par application du schème RE- à (s')habiller : (se) déshabiller / (se) rhabiller. Mais on n'a pas le même type de rapport d'antonymie entre ( $\left.s^{\prime}\right)$ habiller et (se) redéshabiller, car ce dernier verbe n'a pas d'acception annulative mais seulement une acception itérative.

L'item (se) rhabiller peut servir à exprimer aussi bien une valeur annulative qu'une valeur itérative. Il est, de ce point de vue, polysémique. Mais un moyen constructionnel simple de lui conférer une valeur exclusivement itérative est de substituer la variante re- à la variante $r$-: contrairement à (se) rhabiller, (se) rehabiller n'est interprétable qu'itérativement $^{18}$. Or, il se trouve qu'il s'agit là d'un phénomène très général, systématiquement lié à la variante re-. On peut s'en convaincre en examinant la liste suivante, qui ne donne qu'un tout petit échantillon de cette régularité.

(23) (a)

raccompagner

racheter

réactiver

réadapter

réanimer

réapprendre

réapprovisionner

réarmer

réhydrater

réinsérer

réorienter (b)

reaccompagner
reacheter
reactiver
readapter
reanimer
reapprendre
reapprovisionner
rearmer
rehydrater
reinsérer
reorienter

Dans chacun de ces couples, le terme de la colonne (23a) a une valeur annulative, éventuellement doublée d'une valeur itérative. Mais son « doublon » de la colonne (23b) n'a, lui, qu'une valeur itérative. Tout se passe donc comme si la variante re-du morphème $R E-$ avait pour effet général de sélectionner, parmi les différents effets de sens de cette opération, un effet purement itératif quand elle s'applique à une base à initiale vocalique. Ou, pour reprendre la terminologie utilisée plus haut, tout se passe comme si la variante resélectionnait comme argument du foncteur " à nouveau $(x)$ » un procès, un événement ou une activité quand la base a un segment initial vocalique.

Cette observation soulève toutes sortes de problèmes au plan théorique. Par exemple, elle conduit à se demander s'il est encore possible, dans ces conditions, de considérer les variantes $r e-, r e ́$ - et $r$ - comme manifestant le même morphème (donc comme des allomorphes). On notera par ailleurs la conséquence suivante, impliquée par notre observation : la variante $r e$ - permet apparemment de sélectionner un procès, un événement ou une activité (d'où un effet de sens systématiquement itératif), mais seulement quand elle prend la place de ré- ou de $r$-, donc seulement devant une base à initiale vocalique ${ }^{19}$. Devant une base à initiale consonantique, où elle apparaît " régulièrement », cette sélection est par définition impossible. Ainsi, le verbe reboucher est interprétable itérativement ou annulativement ; mais, parce qu'il comporte déjà la variante re-, il n'est pas possible de lui faire subir la même transformation qu'aux items de (23a) ! On a donc là un schème constructionnel qui permet de réduire certains faits de polysémie, mais seulement dans des environnements phonologiques spécifiques.

\footnotetext{
${ }^{18}$ Peu importe évidemment ici l'orthographe (avec ou sans trait d'union).

${ }^{19}$ Avec quelques très rares exceptions, comme réchauffer / rechauffer ou réviser / reviser.
} 
En réalité, cette situation n'est pas complètement exceptionnelle. Un autre schème constructionnel en français - également lié à la négation ! - présente un tableau de phénomènes ressemblant étrangement à ce qui vient d'être décrit (cf. Apothéloz 2003 et 2005). Il s'agit de la préfixation $I N-$. Ce morphème est généralement décrit comme présentant trois variantes allomorphiques : [in-] devant une voyelle, [ $\tilde{\varepsilon}-]$ devant une consonne nonsonante, et [i-] devant une consonne sonante ${ }^{20}$. Toutefois, on s'aperçoit que cette distribution est elle-même sujette à variation : devant une consonne sonante, on rencontre en effet tantôt [i-] tantôt [ $\tilde{\varepsilon}-]$, comme si le système oscillait entre une allomorphie à 2 variantes ([in-] devant voyelle, $[\tilde{\varepsilon}-]$ devant consonne) et une allomorphie à 3 variantes (celle décrite ci-dessus, qui distingue deux classes de consonnes). Par ailleurs, une analyse sémantique montre que devant une consonne sonante, il n'est pas sûr du tout qu'on ait affaire au même morphème. Du moins le sens des lexèmes ainsi construits permet-il d'en douter. Examinons les séries suivantes :

(24) (a)

irrécupérable irréparable irrespirable irremplaçable etc. (b)

inrécupérable inréparable inrespirable inremplaçable

On s'aperçoit que la variante [i-] de la colonne (24a) est systématiquement associée à des lexèmes dont le sens (ou l'un des sens) est superlatif. Comme l'a observé Gaatone (1987), cette superlativité s'accompagne d'une attrition plus ou moins complète du sens négatif : irréparable dans son acception superlative signifie quelque chose comme " définitif », et non pas "qui ne peut être réparé ». Mais la variante [ $\tilde{\varepsilon}-]$ de (24b) est, elle, systématiquement associée à des lexèmes dont le sens négatif, compositionnel, est intact. Cette restauration d'un sens compositionnel est impossible quand la base commence par une consonne non-sonante, puisque $[\tilde{\varepsilon}-]$ est alors l'allomorphe régulièrement sélectionné. C'est ainsi que irremplaçable, qui a des emplois compositionnels avec une négation pleinement présente ("qu'on ne peut pas remplacer ») et des emplois superlatifs avec attrition de la négation (" extrêmement précieux, unique »), peut être reconstruit comme adjectif négatif avec inremplaçable ; mais cette même restauration est impossible avec un adjectif comme incroyable ! C'est la raison pour laquelle nous avons qualifié [ $\tilde{\varepsilon}-]$ devant consonne sonante de morphème « opportuniste » (Apothéloz 2003, 2005).

On le constate, le parallélisme des deux situations, celle de $R E$ - et celle de $I N-$, est quasi parfait! Dans les deux cas on est amené à envisager l'hypothèse qu'il existe deux morphèmes préfixaux distincts, dont l'un est un morphème opportuniste :

- concernant $R E-$ : un morphème $R E_{1^{-}}$se manifestant par trois allomorphes (ré- et $r$-, devant une base à initiale vocalique, et $r e$ - devant une base à initiale consonantique); et un morphème $R E_{2}-\mathrm{n}$ 'intervenant que sur des bases dont le segment initial est une voyelle, et produisant une itération de procès, d'événement ou d'activité (à l'exclusion de tout effet annulatif). Ce second morphème est manifesté par un seul morphe : re-.

- concernant $I N$ - : un morphème $I N_{I^{-}}$se manifestant par trois allomorphes ([in-] devant une voyelle, [ $\tilde{\varepsilon}-]$ devant une consonne non-sonante, et [i-] devant une consonne sonante), qui

\footnotetext{
${ }^{20}$ Sont sonantes les consonnes [B], [1] ainsi que les consonnes nasales. Toutes les autres consonnes sont nonsonantes. En raison du décalage entre la notation orthographique et la réalisation phonétique, nous donnons ici ces variantes en alphabet phonétique.
} 
construit des lexèmes à sens négatif pouvant secondairement dériver un sens superlatif ; et un morphème $I N_{2}-$ n'intervenant que sur des bases dont le segment initial est une consonne sonante, et produisant un lexème à valeur exclusivement négative. Ici également ce second morphème n'a pas d'allomorphie, sa réalisation étant toujours [ $\widetilde{\varepsilon}-]$.

\section{2. $R E$ - et les temps composés}

Un autre problème méritant examen est celui du comportement de $R E$ - aux temps composés. Faute de place, nous n'en ferons ici qu'un exposé extrêmement sommaire. Le problème est le suivant.

On rencontre, dans l'oral conversationnel ordinaire, des formes verbales fléchies à un temps composé dans lesquelles $R E$ - est concaténé non pas au verbe lexical mais au verbe auxiliaire : je resuis sorti, nous resommes partis, ils resont tombés, etc ${ }^{21}$. Comme le verbe lexical peut avoir lui-même lexicalisé un dérivé en $R E-$, il en résulte, une fois encore, la possibilité de doublons, selon que le préfixe est concaténé au verbe lexical ou à l'auxiliaire :

(25) (a)

je suis ressorti nous sommes repartis ils sont retombés je suis redescendu (b)

je resuis sorti nous resommes partis ils resont tombés je resuis descendu

Or, on constate ici aussi que ces variantes de formulation n'ont pas les mêmes interprétations sémantiques. Chaque fois que le verbe lexical en $R E-\mathrm{a}$, ou peut avoir, un sens annulatif - ce qui est le cas des quatre exemples (25a) - le doublon consistant à concaténer le préfixe à l'auxiliaire n'a quant à lui qu'un sens itératif. Ainsi, je suis ressorti peut servir à produire les deux effets de sens « à nouveau je suis sorti » (itératif) et " je suis sorti après être entré » (annulatif), mais je resuis sorti ne peut en principe servir qu'à signifier "à nouveau je suis sorti ». De même ils sont retombés produit les sens "à nouveau ils sont tombés » et "ils sont tombés après s'être (re)levés », mais ils resont tombés ne produit que le sens "à nouveau ils sont tombés ».

Ces phénomènes mériteraient évidemment d'être soigneusement documentés. Il apparaît toutefois qu'on est en présence, ici encore, de la distinction entre $R E_{1^{-}}$(25a) et $R E_{2^{-}}(25 \mathrm{~b})$, quoique les conditionnements ne soient pas les mêmes que plus haut : dans les exemples de type réinsérer vs reinsérer, $R E_{2}$ - était limité à des bases à initiale vocalique ; ici la limitation concerne le statut grammatical de la forme affectée (auxiliaire de conjugaison) ${ }^{22}$.

\subsection{Affixes primaires et affixes secondaires}

En somme, les analyses qui précèdent aboutissent à des conclusions paradoxales. D'une part, sur le plan sémantique, de nombreux faits montrent que la préfixation en $R E$ - gagne à être décrite comme la marque segmentale d'un seul et même schème constructionnel. Mais d'autre

\footnotetext{
${ }^{21}$ D'où, pour le verbe auxiliaire, des faits de défectivité : des formes comme je resuis, nous resommes, il resera, etc., relativement fréquentes, n'ont de toute évidence pas le même statut lexical ou mémoriel que re-être (ou rêtre), il re-est (ou il rest), etc.

${ }^{22}$ A cela s'ajoute peut-être une autre différence sémantique : il semble que les passés composés dans (25a) puissent être interprétés, comme c'est normalement le cas pour ce tiroir verbal, soit comme des accomplis du présent soit comme des perfectifs du passé ; tandis que leur équivalent de la colonne (25b) ne peut être interprété que comme un perfectif du passé ! On aurait là un indice supplémentaire des nombreuses interférences entre $R E$ et la catégorie de l'aspect.
} 
part, sur le plan formel, les faits paraissent plaider pour l'existence de deux schèmes distincts, l'un d'eux (celui représenté par le morphème opportuniste) étant restreint à des environnements morphophonologiques spécifiques.

Quoi qu'il en soit, il semble que les fonctionnements que ces analyses mettent en évidence correspondent à ce qu'on appelle parfois, depuis Bloomfield (1933), affixes primaires (ou de classe I) et affixes secondaires (ou de classe II) ( $R E_{1^{-}}$et $I N_{l^{-}}$-étant des affixes primaires, $R E_{2}$ - et $I N_{2}$ - des affixes secondaires). Depuis les travaux de Bloomfield ces notions ont été redéfinies de diverses manières, notamment dans le cadre de la phonologie générative. Elles correspondent pour l'essentiel aux faits suivants (voir Aronoff 1976, Anderson 1992, Katamba 1993) :

- Les affixes primaires et les affixes secondaires se distinguent par les propriétés prosodiques et/ou morphophonologiques des lexèmes qu'ils construisent. En anglais par exemple, les affixes primaires modifient la place de l'accent principal de la base à laquelle ils se concatènent, alors que les affixes secondaires ne modifient pas la place de cet accent. Ainsi le suffixe -ness est un affixe secondaire (cf. serious, seriousness : ['siərıəs], ['siəriəsnis]), tandis que -ic est un affixe primaire (cf. democrat, democratic : ['deməkræt], [demə'krætık]). En français, $R E_{1^{-}}$et $R E_{2^{-}}$, et $I N_{1^{-}}$et $I N_{2^{-}}$, se distinguent eux aussi par leurs propriétés morphophonologiques : alors que la variante secondaire peut produire, à la frontière du préfixe et de la base, des séquences phonologiques qui ne se rencontrent normalement pas à

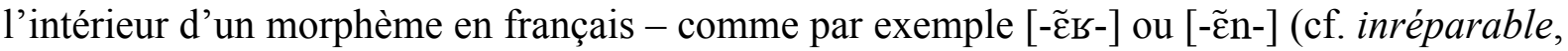

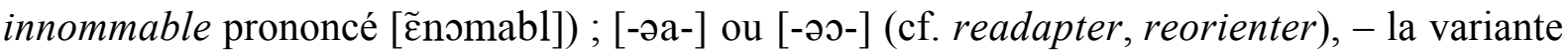
primaire produit quant à elle des séquences conformes aux régularités phonologiques de la langue (irréparable, innommable prononcé [inomabl], réadapter, réorienter).

- Les affixes secondaires sont les plus « externes » de la structure du dérivé. De là, probablement, le fait qu'ils puissent parfois être séparés de leur base. Par exemple, ce que nous avons observé à propos des temps composés signifie que $R E_{2}$ - peut être utilisé en facteur commun de plusieurs verbes lexicaux (je resuis descendu et tombé, avec le sens « je resuis descendu et je resuis tombé $\gg)^{23}$. De là aussi, s'agissant de $R E_{2^{-}}$, l'analogie avec le fonctionnement d'un adverbe. L'idée générale est donc ici que les affixes primaires interviennent à un niveau plus profond de la structure du construit, que ne le font les affixes secondaires. Quand ils sont composés l'un sur l'autre, formant un tandem de préfixes, le préfixe secondaire est toujours le plus externe (cf. reréactiver, reréapprendre, reraccompagner, etc.). Sémantiquement, cela signifie que les valeurs de type (iv) (effet de sens annulatif) interviennent toujours, dans l'histoire dérivationnelle du lexème construit, avant les valeurs des types (i), (ii) et (iii).

- Les affixes secondaires produisent en général un construit dont le sens est davantage compositionnel que les affixes primaires (cf. inréparable vs irréparable, reaccompagner vs raccompagner). Ainsi les dérivés (23b) et (24b) sont davantage compositionnels que les dérivés (23a) et (24a). Par rapport à ce que nous avons écrit au $\S 1.2$. à propos du mode de traitement des lexèmes construits, on peut donc faire l'hypothèse que des lexèmes comme inréparable et readapter seront préférentiellement traités par activation d'un schème constructionnel, tandis que irréparable et réadapter seront préférentiellement traités par accès mémoriel direct. Autrement dit les premiers sont moins lexicalisés que les seconds, au sens (d) défini plus haut ${ }^{24}$.

- Cette différence de compositionnalité sémantique peut se manifester de diverses manières. S'agissant de verbes, les propriétés actancielles sont tout particulièrement

\footnotetext{
${ }^{23}$ Il s'agit de ce que Mel'čuk (1993) nomme « tmèse coordinative ».

${ }^{24}$ On pourrait dire à cet égard que le phénomène de morphème opportuniste atteste de la constante pression des mécanismes constructionnels sur les mécanismes mémoriels.
} 
concernées. Par exemple, les affixes primaires peuvent modifier les propriétés actancielles de leur base, ce qui n'est pas (ou moins) le cas des affixes secondaires. Pour le faire voir comparons irremplaçable et inremplaçable. L'adjectif remplaçable hérite normalement des propriétés actancielles du verbe dont il dérive (d'où : X est remplaçable par Y). Mais, alors que cette propriété est également héritée avec l'affixe négatif secondaire ( $X$ est inremplaçable par Y), elle disparaît pratiquement avec l'affixe négatif primaire (? X est irremplaçable par $Y)$. Dans cet exemple, la perte des propriétés actancielles est évidemment liée à la prégnance de la valeur superlative de irremplaçable.

\section{Bilan et conclusion}

Cette étude des aspects morpho-sémantiques, lexicaux et morphophonologiques de la préfixation $R E$ - a révélé l'extrême complexité de ce schème constructionnel.

En ce qui concerne ses valeurs sémantiques, notre approche a été de postuler que $R E$ peut être ramené, par-delà les nombreux effets de sens qu'il produit, à un unique foncteur mono-argumental, intuitivement représentable par la glose " à nouveau $(x)$ ». La diversité des valeurs sémantiques qu'il produit dans le lexique s'explique alors par le type de l'information susceptible d'instancier la variable $x$ de ce foncteur.

L'examen de quelques cas types nous a conduit à distinguer au moins quatre situations, que nous avons nommées : (i) itération du procès, (ii) itération de l'événement, (iii) itération de l'activité, et (iv) itération d'un paramètre du verbe base. Ces quatre situations rendent compte des deux classes majeures d'effets de sens engendrés par cette préfixation : les sens itératifs (situations (i), (ii) et (iii)) et les sens annulatifs (situation (iv)). La situation (iv) est particulièrement intéressante, car elle montre que du point de vue sémantique, les opérations constructionnelles n'opèrent pas toujours sur un donné qui émane directement de la base. Bien au contraire, ce sur quoi opère $R E$ - dans la situation (iv) est une information qu'il s'agit d'inférer à partir du sens du verbe base. Nous avons appelé cette information un " paramètre » de la base.

Par ailleurs, certains construits lexicaux confirment l'hypothèse d'une valeur unique de $R E$-. Il s'agit de lexèmes apparemment construits au moyen de cette préfixation, mais dans lesquels le segment préfixé ne fait que sanctionner - telle est du moins notre hypothèse - un composant de sens déjà présent dans la base. Ce phénomène de "morphémisation » aboutit à créer des doublons sémantiques.

$\mathrm{Du}$ point de vue du lexique, le schème constructionnel marqué par $R E$ - est en partie associé à la relation d'antonymie directionnelle. A partir de l'analyse des micro-systèmes lexicaux engendrés par cette relation, nous avons montré qu'on doit distinguer deux types d'antonymes directionnels, que nous avons nommés " antonymes lexicaux » et « antonymes discursifs ». Seuls les antonymes discursifs (type monter vs redescendre) dépendent de RE-. Ils sont discursifs en ceci qu'ils comportent une dimension intrinsèquement anaphorique. $\mathrm{Ce}$ lien entre les opérations constructionnelles et l'organisation du lexique nous a également donné l'occasion de distinguer quatre type de processus de lexicalisation.

Du point de vue de ses caractéristiques morphophonologiques, nous avons observé que $R E$ - présente un double fait variationnel : d'une part une allomorphie au sens classique du terme, avec distribution (quasi) complémentaire ; d'autre part, un phénomène de morphème " opportuniste », n'apparaissant que dans un environnement morphophonologique particulier : une base débutant par une voyelle. Ce morphème présente la caractéristique d'exclure les valeurs annulatives. Cet ensemble de propriétés, qu'on retrouve presque à l'identique dans la préfixation négative $I N$-, amène à conclure à l'existence de deux morphèmes $R E$-. L'analyse morphophonologique de $R E$ - conduit donc, paradoxalement, à des 
conclusions inverses de son analyse sémantique. Ces deux variantes de $R E$ - correspondent à ce qu'on appelle parfois, en morphologie constructionnelle, "affixe primaire " et " affixe secondaire $»$.

\section{Références}

Ajdukiewicz, Kazimierz (1967). Syntactic connexion. In : S. Mc Call (ed.), Polish logic, Oxford : Clarendon Press, 207-231.

Amiot, Dany (2002). Re-, préfixe aspectuel ? In : V. Lagae, A. Carlier \& C. Benninger (textes réunis par), Temps et aspect : de la grammaire au lexique. Amsterdam : Rodopi, 1-20. (Cahiers Chronos 10)

Anderson, Stephen R. (1992). A-Morphous Morphology. Cambridge : Cambridge University Press.

Apothéloz, Denis (2002). La construction du lexique français. Paris/Gap : Ophrys.

Apothéloz, Denis (2003). Le rôle de l'iconicité constructionnelle dans le fonctionnement du préfixe négatif IN-. Cahiers de Linguistique Analogique, ${ }^{\circ}$ 1, 35-63.

Apothéloz, Denis (2005). Morphème opportuniste et lexicalisation d'inférences : la préfixation négative $I N$-. Neophilologica, 17, sous presse (Katowice, Université de Silésie).

Aronoff, Mark (1976). Word Formation in Generative Grammar. Cambridge, Mass. : The MIT Press.

Beard, Robert (1990). The nature and origins of derivational polysemy. Lingua, $\mathrm{n}^{\mathrm{o}}$ 81, 101140.

Berrendonner, Alain \& Viviane Clavier (1997). Examen d'une série morphologique dite "improductive" en français : les noms dénominaux en -age. Silexicales, n 1, 35-44.

Bloomfield, Leonard (1933). Language. New York : Holt, Rinehart \& Winston. (Trad. franç. : Le langage. Paris : Payot, 1970)

Corbin, Danièle (1989). Contraintes et création lexicales en français. L'Information Grammaticale, 42, 35-43.

Dell, François (1979). La morphologie dérivationnelle du français et l'organisation de la composante lexicale en grammaire générative. Revue Romane, XIV, 2, 185-216.

Fradin, Bernard (2003). Nouvelles approches en morphologie. Paris : Presses Universitaires de France.

Gaatone, David (1987). Les préfixes négatifs avec les adjectifs et les noms verbaux. Cahiers de lexicologie, 50/1, 79-90.

Guilbert, Louis (1986). L'affixe r-, re- ou ré-. Grand Larousse de la langue française, 48184819.

Jalenques, Pierre (2001). Quand la diachronie renvoie à la synchronie : étude des emplois idiomatiques du préfixe re en français (renier, remarquer, regarder, etc.). Recherches linguistiques de Vincennes, 30, 39-61.

Jespersen, Otto (1922). Language, its nature, development and origin. Londres : George Allen \& Unwin, Ltd. Trad. franç. : Nature, évolution et origines du langage, Paris, Payot, 1976.

Katamba, Francis (1993). Morphology. Houndmills : Palgrave.

Léger, Jean (1956). A propos du préfixe re-. Le Français moderne, 24, nº 4, 285-290.

Léger, Jean (1957). A propos du préfixe re- (notes complémentaires). Le Français moderne, $25, \mathrm{n}^{\mathrm{o}} 1,125-126$.

Lehmann, Christian (1995). Thoughts on Grammaticalisation. München : Lincom Europa.

Lyons, John (1977). Semantics, I. Cambridge : Cambridge University Press. 
Mascherin, Laurent (à paraître). Aspect lexical, affixe et compositionnalité.

Mel'čuk, Igor (1993). Cours de morphologie générale, vol. 1. Montréal : Les Presses de l'Université de Montréal / CNRS Editions.

Mok, Q.I.M. (1964). Le préfixe re- en français moderne ; essai d'une description synchronique. Neophilologus, 48, 96-114.

Mok, Q.I.M. (1980). Le préfixe re- re-regardé : productivité et potentialité. In : D.-J. Van Alkemade, A. Feitsma, W.J. Meys, P. van Reenen, J.J. Spa (eds), Linguistic Studies Offered to Berthe Siertsema. Amsterdam : Rodopi, 205-216.

Rey, Alain (sous la dir. de) (1992). Dictionnaire historique de la langue française, 2 vol. Paris : Le Robert.

Roché, Michel (à paraître). Quelques exemples de morphologie non conventionnelle dans les formations construites à partir d'un mot en -ouille(r). In : B. Fradin (éd.), La raison morphologique. Hommage à Danièle Corbin. Amsterdam : John Benjamins.

$T L F i=$ Trésor de la langue française informatisé. Site internet: $<$ http://zeus.inalf.fr/tlfv3.htm> 